ARTÍCULO

\title{
Dimensiones epistemológicas en los sistemas de accountability de la calidad educativa: elementos para una interrogación crítica
}

\author{
Carmelo Galioto-Allegral (D) \\ Luís Manuel Flores-Gonzalez'

\section{RESUMEN}

El artículo interroga críticamente las premisas epistemológicas que fundamentan los sistemas de accountability de la calidad educativa. El foco de este ejercicio teórico es la concepción del conocimiento que subyace en los indicadores de la calidad educativa, con la finalidad de discutir sus principios y límites fundamentales. En la primera parte, y tomando como referencia el caso chileno, desarrollamos una descripción general de la accountability, y de los supuestos epistemológicos que justifican su coherencia interna, así como de una supuesta objetividad, referida a estándares de medición y de rendimiento. En la segunda sección, problematizamos los límites de la noción de objetividad desde enfoques epistemológicos de la física contemporánea y de la fenomenología. En conclusión, planteamos algunas implicancias y lineamientos para las políticas de aseguramiento de la calidad educativa.

PALABRAS CLAVE

calidad educativa; accountability; objetividad; epistemologías de la complejidad.

'Pontificia Universidad Católica de Chile, Santiago, Chile. 


\title{
EPISTEMOLOGICAL DIMENSIONS IN THE ACCOUNTABILITY SYSTEM OF EDUCATIONAL QUALITY: ELEMENTS FOR A CRITICAL INQUIRY
}

\begin{abstract}
The paper critically inquiries the epistemological premises that underpin the accountability systems of educational quality. The focus of this theoretical exercise is the conception of knowledge that underlies the indicators of educational quality, in order to discuss its fundamental principles and limits. In the first part, we developed a general description of accountability and its epistemological assumptions, taking as a reference the Chilean case, that justify its internal coherence, as well as a supposed objectivity, referring to measurement and performance standards. In the second section, we critically discussed this notion of objectivity from epistemological approaches as contemporary physics and phenomenology. In conclusion, we presented some implications and guidelines for quality assurance in educational policies.
\end{abstract}

KEYWORDS

educational quality; accountability; objectivity; epistemology of complexity.

\section{DIMENSÕES EPISTEMOLÓGICAS DO SISTEMA DE ACCOUNTABILITY DA QUALIDADE EDUCATIVA: ELEMENTOS PARA UM QUESTIONAMENTO CRÍTICO}

RESUMO

$\mathrm{O}$ artigo questiona criticamente as premissas epistemológicas que sustentam o sistema de accountability da qualidade educacional. $\mathrm{O}$ foco deste exercício teórico é a concepção de conhecimento subjacente aos indicadores de qualidade educacional a fim de discutir seus princípios e limites fundamentais. $\mathrm{Na}$ primeira parte, e tomando como referência o caso chileno, é desenvolvida uma descrição geral da responsabilidade e dos pressupostos epistemológicos que justificam sua coerência interna, bem como de uma suposta objetividade referente aos padrões de medição e desempenho. Na segunda seção, os limites da noção de objetividade são problematizados a partir de abordagens epistemológicas da Física e da Fenomenologia contemporâneas. Em conclusão, apresentamos algumas implicâncias e orientações às políticas educativas de qualidade educativa.

PALAVRAS-CHAVE

qualidade educacional; accountability; objetividade; epistemologias da complexidade. 


\section{INTRODUCCIÓN}

En este artículo desarrollamos una interrogación crítica de las premisas epistemológicas que fundamentan y justifican los sistemas de accountability como mecanismo de evaluación de la calidad educativa. Para llevar a cabo este ejercicio teórico, tomaremos en cuenta aspectos y elementos procedentes del sistema escolar chileno, abordando la siguiente pregunta ¿Qué problemas epistemológicos encierra el supuesto de la accountability según el cual ciertos datos producidos por las mediciones estandarizadas dan cuenta de la calidad de las instituciones educativas?

Se trata, por un lado, de explorar qué premisas puede exhibir los sistemas de accountability, más allá de los argumentos psicométricos, respecto del saber producido por sus instrumentos, $y$, por otro lado, de problematizar tales premisas, con la finalidad de explicitar las concepciones de conocimiento de la calidad educativa implicadas en estos supuestos; para llevar a cabo este ejercicio teórico, tomaremos en cuenta aspectos y elementos procedentes del sistema escolar chileno.

Ahora bien, los sistemas de school accountability vinculados al aseguramiento de la calidad educativa son elementos medulares de las agendas de governance de la escuela en muchas áreas geográficas desde hace veinte años (Verger, Fontdevila y Parcerisa, 2019). Suficiente literatura permite afirmar, por un lado, que la cuestión de la accountability para la calidad educativa es un tema abordado por múltiples disciplinas de las ciencias sociales (Suspitsyna, 2010; Ydesen, 2013; Clarke, 2014). Por otra parte, la concepción de la calidad educativa ligada a sistemas de accountability se ha consolidado a nivel global. Lo anterior se puede comprobar, por ejemplo, en espacios geopolíticos como la Unión Europea (Grek, 2009; Ozga, 2013), en Estados Unidos con las políticas No child left bebind y Race to the to $p^{1}$ (Chiang, 2009; Hemmer, Madsen y Torres, 2013) y en Australia con un abanico de medidas y reformas (Cumming, van der Kleij y Adie, 2019). Además, hay literatura que explora el impacto del uso de test con altas y bajas consecuencias en China (Chen y Teo, 2020). Además, respecto de la vinculación de la accountability con las políticas de aseguramiento de la calidad educativa, Brasil ha sido un caso estudiado junto con países muy diversos entre sí como son China y Rusia (Kauko, Rinne y Takala, 2018).

Cabe señalar, también, que el Programme for International Student Assessment $(\mathrm{PISA})^{2}$ constituye, según ciertos autores, una pieza clave del régimen de la accountability puesto que establece, para los sistemas escolares, parámetros de comparación

1 Se trata de dos políticas educativas de gran envergadura en términos de gasto fiscal que buscaban la mejora del sistema escolar, mediante un variado set de instrumentos de la accountability; la primera fue implementada por la administración Bush desde el año 2002; la segunda fue anunciada por la administración Obama en 2009.

2 Esta prueba estandarizada es administrada por la Organización para la Cooperación y el Desarrollo Económico (OCDE) desde el año 2000. La prueba PISA podría ser un caso ejemplar respecto el cual deconstruir sus premisas epistemológicas. Los argumentos que desarrollaremos se pueden aplicar por extensión a este test de carácter internacional. 
a nivel internacional (Lingard, Martino y Rezai-Rashti, 2013; Sellar y Lingard, 2014; Lewis y Lingard, 2015) ${ }^{3}$.

Ahora bien, el debate académico en torno a la accountability en educación, desde el ámbito de los policy studies, es uno de los más controvertidos debido a las discusiones sobre los efectos de este mecanismo en los sistemas escolares. El trabajo de Grek (2009) define el efecto de los sistemas de accountability en términos de un pasaje brusco, para las instituciones educativas, desde un trabajo enfocado en la cultura, a un trabajo enfocado en números, logros y resultados: esto marcaría el ingreso en la que el politólogo Michael Power (1997) ha denominado audit society.

Algunas revisiones de literatura en torno a las políticas de evaluación y accountability escolar (Au, 2007; Rosenkvist, 2010; Falabella, 2016a) examinan críticamente los efectos de las pruebas estandarizadas con altas consecuencias en las instituciones educativas, señalando especialmente la aparición del teaching to the test: la enseñanza se reduce a adiestrar en vista de responder a las pruebas, en detrimento de otras dimensiones del curriculum escolar.

Por otra parte, existe un corpus de investigación académica que se concentra en un análisis de la validez técnica de los instrumentos de evaluación en que se apoyan los sistemas de accountability (Crocker, 2002; Cizek, Rosenberg y Koons, 2008; Ortíz, 2012; Flórez, 2013; Brewer, Knoeppel y Lindle, 2015). Esta literatura discute, mediante un variado abanico de argumentos, la validez y confiabilidad (validity and reliability) de los test que acompañan a la accountability. También recuerdan que en rigor las mediciones son siempre aproximaciones y no mediciones directas del fenómeno en cuestión.

Estos argumentos apuntan a la coherencia y consistencia interna de la construcción del test y pueden involucrar aspectos psicométricos/estadísticos de diseño la prueba, así como el modo en que dichas pruebas se refieren respecto de lo que quieren medir. Por ejemplo, si la misma evaluación, en dos tiempos distintos, se efectúa con diversos universos de estudiantes, en ese caso pierde parte de su validez evaluativa; eso porque no está recogiendo eventuales cambios (para bien o para mal) de los mismos estudiantes en el tiempo, sino que evalúa dos grupos de estudiantes diferentes: eso significa que no evalúa una mejora o empeoramiento real respecto a la primera medición efectuada.

Otro campo de investigación respecto de la validez de los sistemas de accountability en educación aborda el aspecto de la consequential validity (Brewer, Knoeppel y Lindle, 2015). Este enfoque plantea que la validez de la accountability se expresa y recoge a partir de la interpretación y uso de los resultados de los test. En palabras de Brewer, Knoeppel y Lindle (2015, p. 721) "cuando los test se utilizan en la toma de decisiones la validez del test está ligada también a su uso y a las consecuencias reales que tiene para las personas" e identifican dos dimensiones de

3 Una pregunta interesante respecto de la difusión y circulación de las políticas de accountability en el ámbito educativo es la siguiente ¿jpor cuales razones se asiste a ese fenómeno a escala global, ¿cuáles son los elementos que ayudan a comprenderlo? Abordar esta pregunta supera los propósitos de este paper. Sin embargo, señalamos un estudio (Verger, Fontdevila y Parcerisa, 2019) que presenta una investigación respecto de esta pregunta. 
este tipo de validez. La primera sugiere que las categorizaciones fruto de los puntajes deberían reflejar cuidadosamente las habilidades y conocimientos evaluados ${ }^{4}$. La segunda dimensión tiene que ver con las consecuencias tanto potenciales como reales de la aplicación del test.

Otros autores han explorado los problemas éticos de los sistemas de accountability (Sellar, 2015b), así como han desarrollado un análisis que enfatiza la angustia de las continuas evaluaciones (Harvey, 2010). Por otra parte, Educational Assessment on Trial (Davis y Winch, 2015) es un caso ejemplar de ejercicio filosófico que pasa al cedazo crítico los test usados como instrumentos de accountability confrontando argumentos diferentes acerca del rol de las pruebas estandarizadas en educación.

El libro de Davis y Winch (2015) junto a algunos papers del mismo Davis $(2006,2013,2015)$ desarrollan una discusión filosófica de los test de medición vinculados al accountability educativo. Las argumentaciones filosóficas de Davis se dirigen al marco de posibilidades lógicas y epistemológicas de los test de llegar a dar cuenta de lo que los estudiantes pueden saber de un contenido curricular. El foco de los análisis de Davis es la consistencia de los test respecto a los aprendizajes esperados, es decir su capacidad evaluativa, a través del desarrollo de argumentos de principio ligados a un enfoque filosófico y no de carácter técnico.

A pesar de esta contundente evidencia empírica acumulada y de los análisis teóricos realizados, es todavía muy incipiente un abordaje epistemológico de los sistemas de accountability en la educación escolar. En efecto, las pruebas estandarizadas y los test producen un conjunto de datos que avanzan una pretensión de conocimiento respecto a los aprendizajes, es decir respecto de lo que ocurre en la relación educativa entre docentes y estudiantes y en general respecto de la experiencia escolar misma (Lacruz, Américo y Carniel, 2019; Albuquerque, Ferreira y Barreira, 2020) La calidad de la escuela sería conocida, recogida y expresada, mediante los sistemas de accountability.

Mostraremos la relevancia y pertinencia de la problematización que queremos desarrollar, a partir del caso chileno que presenta algunas características específicas: una de las principales novedades de las políticas de rendición de cuentas de la calidad en Chile ha sido la creación, en 2011, de la Agencia de la Calidad Educativa: una institución gubernamental que tiene la misión de evaluar, ordenar y orientar a los establecimientos educativos de Chile, encargándose de hecho de gestionar los sistemas de accountability de la calidad educativa (Carrasco, 2013).

Existe un corpus de literatura, en Chile, que ha estudiado críticamente los sistemas de rendición de cuentas de la calidad, preguntando qué tipos de procesos de mejoramiento impulsan (Falabella, 2014, 2016a), analizando en qué tipo de metodología se basa el sistema de clasificación de colegios (San Martín y Carrasco, 2012) y estudiando la dimensión sistémica e ideológica de las políticas de aseguramiento de la calidad (Oliva, 2010; Flórez, 2013, 2015; Falabella, 2016b, 2019).

4 Por ejemplo, un instrumento basado exclusivamente en el IQ induce a pensar que la inteligencia sea algo unidimensional; esta creencia se genera a pesar del reconocimiento de la comunidad científica que la inteligencia es un fenómeno multifacético y multimodal. Se produce así un efecto de reducción respecto de las múltiples características de la inteligencia humana. 
Como profundizaremos en la siguiente sección, Chile representa un caso ejemplar de cómo se ha establecido, en las políticas educativas, una comprensión y concepción de la calidad más amplia respecto al simple resultado de test estandarizados que enfatiza la necesidad de asegurar dicha calidad mediante un control externo a los establecimientos educativos y recurriendo, para lograr este objetivo, a otros indicadores de la calidad.

Por lo tanto, si el foco de atención del consequential validity son las consecuencias de los sistemas de accountability, el foco de análisis del presente artículo es el desarrollo de una interrogación crítica a las premisas y presupuestos de carácter epistemológico que sostienen la vinculación entre calidad educativa y accountability. El tipo de aproximación que desarrollaremos dialoga con el debate suscitado por las contribuciones de Davis, pero se distingue por un interés más marcado respecto a la caracterización y problematización de los presupuestos epistemológicos del conocimiento "objetivo" y estandarizado que rigen la vinculación entre accountability y calidad educativa.

$\mathrm{El}$ artículo procede de la siguiente manera: la primera parte de esta exploración epistemológica consiste en una aproximación general al accountability como dispositivo político. A través de literatura académica sobre el argumento y el recurso a ejemplos recabados desde el instrumento de accountability de Chile llamado Sistema de Medición de la Calidad Educativa (SIMCE) ${ }^{5}$ manejado por la Agencia de la calidad, describiremos las características principales de esta política educativa, haciendo énfasis en el nexo entre calidad, accountability y estandarización: desde esta descripción se identificará la objetividad como rasgo epistemológico distintivo de los varios y distintos procedimientos e instrumentos de evaluación. Después de esta sección descriptiva, discutimos críticamente la noción de objetividad que está a la base de las operacionalizaciones que permiten justificar los procesos de accountability, como un sistema de medición de la calidad escolar dotado de una determinada racionalidad de política educativa. Recurriremos a argumentos procedentes desde la física y de la fenomenología. Finalmente, en las conclusiones, recogemos la respuesta que se perfila respecto de la pregunta que guía este ensayo y se vislumbran algunas implicancias para investigaciones futuras y para las políticas educativas.

\section{APROXIMACIÓN DESCRIPTIVA AL ACCOUNTABILITY EN CUANTO MECANISMO DE POLÍTICA EDUCATIVA}

El school accountability es un tipo de política que surge en el mundo anglosajón ${ }^{6}$ y que se basa en un enfoque que contempla sistemas de evaluación del

5 SIMCE: Sistema de Medición de la Calidad de la Educación. Se inició a implementar en 1988 en cuarto y octavo básico. En su comienzo medía rendimiento en lenguaje y castellano a través de pruebas estandarizadas. Paulatinamente ha ido aumentando las asignaturas evaluadas; este año, por primera vez, la aplicación de esta prueba ha sido suspendida debido a la pandemia por COVID-19.

6 Ydesen (2013, p. 716) recuerda que la primera vez que aparece el término accountability en una ley de política educativa es el 1971. Se trata del Educational Accountability Act of 1971, Section 229.57, Florida Statutes. 
desempeño profesional de los docentes, dispositivos de supervisión y medición de los resultados educativos de los colegios, con el propósito de aumentar la responsabilidad de la escuela hacia padres y estudiantes. Sobre la base de estas mediciones y resultados, se pretende guiar el sistema escolar hacia la mejora de los aprendizajes (Figlio y Ladd, 2020). Conocer los resultados educativos de un establecimiento escolar permite a los profesores conocer el estado de avance de los aprendizajes de sus estudiantes, mientras que las autoridades políticas podrán evaluar el desempeño de los colegios y dirigir de mejor forma los recursos y apoyos pedagógico donde más se necesitan. En este sentido la rendición de cuenta cumple con una función de monitoreo y evaluación del quehacer didáctico de los colegios. Los aspectos problemáticos de la accountability se ligan justamente al uso y propósitos que las políticas educativas puedan hacer de esta herramienta.

El paper "Accountability from a social justice perspective: criticism and proposals" (Flórez y Rozas, 2020) nos ayuda a describir ciertos rasgos característicos de la accountability vinculados a su uso. Se destaca que en sus comienzos las prácticas de accountability querían dar cuenta de asuntos ligados a algo que se poseía, deudas, acuerdos y promesas. Otra característica distintiva de la accountability es además la posibilidad que un actor o institución rinda cuenta a otra entidad. Por lo tanto, sobre la base de esta rendición de cuenta, pueden darse consecuencias, que como veremos, no son siempre satisfactorias, para un desarrollo equilibrado de las instituciones educativas, en que no siempre son medibles dimensiones y procesos no estandarizados.

Las autoras, además, plantean tres formas de accountability: la primera se presenta como una forma burocrática (llamada también jerárquica o administrativa) que consiste en la obligación de responder al marco normativo de los Estados. La segunda forma se concibe en términos de responsabilidad profesional, que se remite como marco de referencia a las normas profesionales que rigen docentes y directivos en las escuelas, y finalmente, una forma con un fuerte énfasis en los resultados que permitan discriminar los diferentes desempeños de las escuelas con el fin de regular un cierto tipo de mercado.

Ahora bien, hay una numerosa literatura académica que plantea que el diseño de los sistemas de accountability ha sido ligada prevalentemente a la evaluación de la calidad educativa, a través de resultados en test estandarizados, y sobre la base de indicadores de desempeño, entregando por lo tanto mayor importancia al rasgo de accountability vinculado al mercado (Sellar y Lingard, 2013, 2014; Ball, 2015; Lingard et al., 2015).

En varios países, entre estos Chile, los resultados negativos en los indicadores de desempeño se ligan a consecuencias para los colegios que, en casos extremos, pueden llegar hasta su cierre. Se trata del mecanismo del high stake tests (Chen y Teo, 2020) ${ }^{7}$ que muestra uno de los rasgos característicos de la accountability, es decir la performatividad (Ball, 2003; Dubnick, 2005; Ball, 2015).

7 Con esta expresión se recoge la asociación de test estandarizados cuyos resultados implican altas consecuencias para los establecimientos educativos. 
Por ejemplo, en Chile, una de las decisiones más relevantes del escenario de las políticas de accountability de los últimos 5 años, ha sido la creación, por parte de la Agencia, de los indicadores de desarrollo personal y social (Chile, 2012,2013, $2015)^{8}$, con el objetivo explícito de avanzar hacia una concepción más amplia de la calidad educativa y medir algunas dimensiones del quehacer educativo de los colegios que antes no eran tomadas en cuenta. Estos indicadores son: clima de convivencia escolar, participación y formación ciudadana, hábitos de vida saludable, asistencia a clase, retención escolar, equidad de género, titulación técnico profesional (Chile, 2017). Dichos indicadores de desarrollo personal y social permiten recabar datos a partir de cuestionarios dirigidos a estudiantes, docentes, padres y apoderados, en el marco de las pruebas SIMCE, donde constituyen un 33\% de la ponderación para clasificar el desempeño del colegio, mientras el otro $67 \%$ lo ocupan los resultados académicos (estos últimos obtenidos mediante test estandarizados). Las categorías son tipificadas en los siguientes niveles de desempeño: alto, medio, medio-bajo e insuficiente. En el caso en que el colegio no logre mejorar la categoría de desempeño insuficiente después de un periodo de tres años, el colegio se cerraría.

Las categorías de desempeño configuran entonces unos estándares de los logros y resultados deseables para los colegios, Por tanto, estos estándares, no son meramente indicativos, sino que pueden implicar sanciones graves.

A la luz de estos rasgos y de la literatura revisada, se puede caracterizar el esquema de la accountability, en el sistema escolar chileno, como un conjunto de regulaciones que pide cuenta a los actores del quehacer educativo en base a ciertos estándares y a categorías de desempeño preestablecidas. Entonces la accountability se ha consolidado en la educación escolar, a través de la aplicación de pruebas estandarizadas y el diseño de indicadores de desempeño ${ }^{9}$ de los colegios; se perfila, de esta manera, un paradigma cuantitativo y objetivo, basado en los datos producidos por las pruebas.

Sellar (2015a) aborda el uso de datos en evaluación de larga escala aclarando algo importante para nuestra interrogación crítica: los datos en el mundo educativo funcionan desde un proceso de commensuration, es decir, se produce una transformación de diferentes cualidades en una métrica común. A su vez, Selwyn (2015) recoge la tesis de Sellar y señala que la producción de los datos implica una hiper-reducción de cualidades éticas y relacionales del fenómeno educativo. A partir de estos antecedentes, podemos plantear que la lógica subyacente a los mecanismos de accountability asume que, una vez que se hayan obtenidos, mediante procedimientos estandarizados, ciertos datos o valores numéricos acerca de un fenómeno $x$ de la educación escolar, estos datos darían cuenta objetivamente, y por lo tanto de manera válida y apropiada, de aquel fenómeno en cuestión.

Además, la objetividad de los datos asume más peso y relevancia puesto que se le asocian mecanismos de sanciones sobre la base de la performance medida por

8 Una descripción de estos indicadores es disponible también en: https://www.agenciaeducacion.cl/evaluaciones/indicadores-desarrollo-personal-social/. Acceso el: 14 abr. 2020.

9 De esta manera dicho sistema de accountability educativo se configura como lo que la literatura ha denominado indicator game (Fochler y De Rijcke, 2017): la calidad de la escuela estaría expresada y reducida a este juego de muchos y diversos indicadores. 
estos dispositivos. Se trata de una lógica dotada de un carácter epistemológico: plantea que el modo de conocer de la accountability es tan válido y objetivo, no sólo porque determina el nivel de conocimiento de logros y aprendizajes de los estudiantes, sino también porque se le otorgar una suerte de poder judicial acerca del quehacer de las escuelas. Es esta lógica epistemológica que ahora vamos a problematizar y discutir.

\title{
DISCUSIONES ACERCA DE LA NOCIÓN DE OBJETIVIDAD
}

\author{
Lo que no se define no se puede medir. \\ Lo que no se mide, no se puede mejorar. Lo que no se mejora, se degrada siempre.
}

(Kelvin, 1824-1907)

Cómo decíamos en la introducción, este paper dirige su perspectiva analítica a los elementos que subyacen a los argumentos psicométricos de validez técnica de la accountability. De esta forma, se exploran las concepciones del conocimiento que se pueden reconocer desde un análisis epistemológico de los presupuestos del nexo entre test estandarizados, resultados medidos y calidad educativa.

Este nexo se funda en la pretensión que la información, que aportan los sistemas de medición en general y de la accountability en particular, permiten establecer un juicio fundado acerca del desempeño "real" de las escuelas. Finalmente, la definición del nexo entre la accountability y calidad educativa planteado en la sección anterior se puede complementar con otro aspecto: la referida a la carrera por los resultados, que es motivada por un imperativo llamado a la mejora continua del sistema educativo. Las instituciones educativas son empujadas hacia un horizonte que se mueve siempre un poco más allá y que lleva a buscar revisiones, cambios e innovaciones pedagógicas. Esta lógica se puede reconducir a una frase que es frecuente oír en las discusiones en torno al accountability en educación: lo que no se mide no se puede mejorar.

Ahora bien, la afirmación lo que no se puede medir no se puede mejorar es la versión simplificada de la afirmación del físico británico Kelvin: lo que no se define no se puede medir. Lo que no se mide, no se puede mejorar. Lo que no se mejora, se degrada siempre. Se trata de una proposición que, a pesar de su evidencia y naturalización, tiene varios reparos y observaciones que provienen incluso de la misma ciencia "natural" y no sólo de la filosofía.

Empezaremos la discusión crítica de la objetividad de la accountability para la calidad educativa, analizando las implicancias de esta frase que proviene del mundo de la física.

Kelvin, (1824-1907) es el científico que pone nombre a la escala de temperatura Kelvin. Esta escala establece el cero absoluto, $\left(-273,15^{\circ} \mathrm{C}\right)$ que, según Kelvin, representa la temperatura más baja posible en el momento que las partículas, carecen de movimiento porque están congeladas. 
En rigor, esta temperatura es inalcanzable en la "realidad", porque es un límite matemático, que permite, como siempre con las escalas, establecer una referencia, en un rango que nos permite medir, para justamente aproximarnos al fenómeno, pero en términos indicativos, y no necesariamente "objetivos" de la materia, o de un fenómeno de la naturaleza. Por ejemplo, las escalas de temperatura, donde el valor cero es una más bien una convención y una referencia, que un criterio "objetivo" de realidad.

A este respecto, son significativos los ejemplos de las escalas de Mercalli y Richter: ambas escalas fueron modificadas, por la intensidad de los terremotos, que sobrepasaron el límite establecido, en sus formulaciones originales.

En el caso de la escala de Richter, se mide la magnitud de la energía liberada de los diferentes sismos. Se le confunde a veces con la escala que mide la intensidad a la que se refiere la escala de Mercalli. La magnitud de un sismo está relacionada con la energía liberada por un temblor o terremoto. El punto de referencia de la escala de Richter corresponde al registro obtenido de un sismógrafo, establecido a una distancia dada del lugar del epicentro. La matematización que realizó Richter (1935), se expresa en una escala logarítmica, en los siguientes términos: "La magnitud $\mathrm{M}$ está relacionada con la energía $\mathrm{E}$ (ergios) liberada en el foco del terremoto por la fórmula aproximativa: $\log \mathrm{E}$ (ergs)=11,4+1,5 M" (Gaulon y Rothé, 2016).

Es relevante señalar, a favor de la línea argumental de este artículo, que esta fórmula es "aproximada" a pesar de que las constantes de la naturaleza, que se expresan matemáticamente, pretendan formular el carácter "universal" de las cosas y de la naturaleza. Es evidente que la escala logarítmica propiamente tal no cambia y los logaritmos, en base 10, siguen y seguirán siendo los mismos. Sin embargo, la cuestión de fondo de relevar aquí es que el criterio que usa toda escala es "relativo" a los pliegues y sutilezas del fenómeno, y, por tanto, lo medido es también relativo. Esto significa, que la escala es parte de una información a nuestras propias medidas, y no corresponde necesariamente a la variedad del fenómeno.

La escala de Richter originalmente medía hasta 6 grados, y fue modificada por Hanks y Kanamori (1979) que formularon una escala de magnitud del momento. En otros términos, la escala de Mercalli igual fue modifica. Ha habido, en estos casos, y en otros, modificaciones importantes, que muestran incontestablemente, la relatividad de las fórmulas no porque estas sean arbitrarias, o estén mal formuladas, sino porque el fenómeno implica siempre una "realidad" más sutil y compleja, que escapa a cualquier medición incluida la más rigurosa ${ }^{10}$.

10 Hasta el momento, la mayor magnitud registrada en el mundo corresponde a 9,5 grados Richter, para el terremoto que ocurrió en Valdivia, Chile, en 1960. La escala Mercalli también modifica (MM), se refiere a la percepción subjetiva de un sismo. Originalmente se establecía un rango de I a X, y se modificó hasta XII, siendo I lo más leve, y XII el nivel catastrófico de un evento telúrico. Este ejemplo es relevante por el intento de medir también la percepción subjetiva de un fenómeno. Por otra parte, en ambos casos, es importante destacar que las medidas de las escalas son las que se adaptan a la "naturaleza" del fenómeno, y no al revés como parece ocurrir a veces en ciencias sociales y particularmente en educación. 
Estos ejemplos son relevantes por el hecho que las ciencias naturales y la física particularmente, establecen leyes, es decir constantes, supuestamente "objetivas" de la realidad, y son estas mismas ciencias, que ponen en cuestión el criterio absoluto, y literalmente "relativizan" tales medidas, y también las supuestas condiciones absolutas de lo que denominamos lo real o realidad (Penrose, 2007, p. 1375).

La física de Einstein, por otra parte, es justamente una respuesta a la medida del valor absoluto del espacio y el tiempo de la física de Newton, y a la vez una "relativización" de la geometría euclidiana. El tiempo como el espacio son relativos, y ambos son correspondientes a la materia. Así, por ejemplo, se puede entender al espacio como curvo, pero no es en sí mismo curvo, sino en relación con la materia, y al movimiento de la misma materia, en el espacio. La necesidad de reformular esta física le permite a Einstein de entrada, no sólo relativizar las proposiciones geométricas, sino además de calificarlas como proposiciones "bastantes imperfectas" cuando se trata de dar cuenta de lo real (Einstein, 2012).

Por tanto, la "objetividad" es puesta entre paréntesis, no sólo por el constructivismo pedagógico, o la biología en clave de Humberto Maturana (1995, 2020), sino por el nacimiento de una epistemología en el ámbito de las ciencias físicas, y de la filosofía que la precede.

La lógica epistemológica de la accountability, que hemos descrito en la sección anterior, parece transitar al margen de estas posiciones, presuponiendo más bien una visión lineal del conocimiento ${ }^{11}$. La cuestión de la accountability, por lo tanto, opera al margen de estas consideraciones, y en cierta forma es causa y consecuencia de una política educativa neoliberal en el orden social, y de una epistemología de la objetividad en el dominio el conocimiento. Esta epistemología concibe una adecuación entre los sujetos, las cosas, y el mundo, que permite establecer sistemas de validación de aprendizajes y de calidad educativa en términos de registros cuantitativos y de performance.

Revisemos ahora la necesidad de elaborar desplazamientos de una epistemología de la objetividad, a una epistemología de la complejidad, y de reconocer sus implicancias respecto a los modos de conocer de los sistemas de accountability (Flores-González, 2008). Este desplazamiento epistemológico puede nutrirse de al menos dos fuentes. Por un lado, la física de Heisenberg que nos proporciona una epistemología de incertidumbre; y por otro, la fenomenología de Merleau-Ponty que desarrolla una aproximación a la epistemología desde lo que el filósofo francés llama el mundo de la vida (Lebenswelt). En ambas visiones hay una crítica a la idea de concebir una realidad objetiva, radicalmente independiente del sujeto o concebida por "sí misma" como en una perspectiva absoluta, es decir liberada de cualquier condicionamiento.

11 Este presupuesto epistemológico se ve reflejado en otros aspectos del sistema escolar, por ejemplo, cuando, basados en una geometría euclidiana, se diseñan abigarrados sistemas curriculares, que configuran el espacio y el tiempo en la escuela (Flores y Marini, 2018). 


\section{EPISTEMOLOGÍA DE LA INCERTIDUMBRE}

Comenzamos con aproximarnos a la epistemología de la incertidumbre planteada por Heisenberg (1901-1976). Este físico fue él que planteó el principio de indeterminación: el conocimiento que tenemos de la materia, en el caso específico de las partículas, no les pertenecen a las partículas en sí mismas, sino del conocimiento que nosotros tenemos de ellas "Resulta de ello, en definitiva, que las leyes naturales que se formulan matemáticamente en la teoría cuántica no se refieren ya a las partículas elementales en sí, sino al conocimiento que nosotros tenemos de ellas" (Heisenberg, 1986, p. 12-13).

Las leyes naturales, los patrones matemáticos, las estadísticas no le pertenecen entonces al objeto estudiado, sino a la información que "explica", pero muy tangencialmente, la "realidad" de un fenómeno determinado. Por ejemplo, el "año normal" de lluvias, que se calcula por el promedio matemático de precipitaciones registradas, definitivamente no existe.

Análogamente, se podría aplicar esta lógica respecto de aspectos que los indicadores de desarrollo personal y social propuestos por la Agencia de la calidad: ¿Existirá la convivencia escolar ideal y normal, calculada por el valor numérico que proporciona dicho indicador?

Efectivamente, si ya en el mundo de la física, hay por así decir una inadecuación estructural entre la medida y lo medido, se pone el problema respecto del alcance y pertinencia de estos criterios cuantitativos y estandarizados para conocer los fenómenos de las ciencias sociales y, en nuestro caso, de la educación.

Por ejemplo, la noción de "estándares", se ha convertido en una dimensión clave que el sistema de accountability integra como parte significativa de la medida de la calidad, no solo de los aprendizajes académicos, sino de otros aspectos como la convivencia escolar y la formación ciudadana. Lo de estándar es un concepto que tiene su origen en un hecho bélico, con fecha precisa y evento determinado. Normand (2005) indica que, según el Diccionario Inglés de Oxford, "el término fue utilizado por primera vez el 22 de agosto de 1138, en referencia a la Batalla del Estándar, una batalla entre los ingleses y los escoceses en Northallerton. El standard correspondía al mástil de un barco con banderas alrededor de las cuales se agrupaban los ingleses. Se llamó estándar porque "fue allí donde el coraje tomó posición para ganar o morir" (Normand, 2005, p. 68-69).

Si ya el significado etimológico es siempre relevante en la historia de una cuestión o problema de carácter teórico y social, en este caso el referente histórico es quizás más decisivo, porque nos da el contexto. Por extensión entonces, el cumplimiento de estándares es sin duda la adquisición de un logro, pero en una competencia que no necesariamente es de iguales, ni en la que evidentemente todos ganen (Ramos, 2018, p. 52).

Esto es relevante de destacar, tanto en el caso de la educación, pero también, en otras áreas, como por ejemplo la economía, en indicadores como el PIB, donde esta lógica de "objetividad", se ejerce sobre principios simples y reduccionistas. Así lo muestran varios trabajos recientes del ámbito de la economía (Piketty y Zucman, 2013; Piketty, 2014) y de estudios de las externalidades que omiten el PIB y de 
otros indicadores en el ámbito del medio ambiente y de la planificación estratégica de las ciudades (Barton y Kopfmuller, 2016) ${ }^{12}$.

Lo anterior implicaría una lógica de guerra y competencia, o si se quiere emplear una metáfora no bélica y no mercantil, se trata de una lógica de una carrera, donde no todos pueden llegar a la meta establecida. Asumir esta lógica en educación y en el mundo de la escuela implica consecuencias: la desvalorización de la escuela como lugar libre de la necesidad de producción y de dinámicas de mercado o económicas.

\section{EPISTEMOLOGÍA DEL MUNDO DE LA VIDA}

Después de haber explorado la pertinencia de la epistemología de la incertidumbre en la cuestión de la accountability de la calidad educativa, es útil recurrir ahora a algunos argumentos de la fenomenología de Merleau-Ponty para comprender aún más los aspectos problemáticos de la lógica de objetividad que subyace a la asociación entre mediciones, estándares de desempeño y calidad educativa.

El fenomenólogo francés desarrolla una crítica profunda a la idea tradicional de ciencia positiva. La ciencia literalmente deja afuera al protagonista del conocimiento: el sujeto que conoce. La ciencia, con su anhelo a la exactitud, se desprende desde la experiencia del mundo que Merleau-Ponty llama mundo de la vida: puesto que la ciencia sería una derivada de segundo orden del mundo de la vida, quedará siempre con un desajuste respecto de esa dimensión primera de la experiencia del mundo percibido:

Todo cuanto sé del mundo, incluso lo sabido por ciencia, lo sé a partir de una visión más o de una experiencia del mundo sin la cual nada significarían los símbolos de la ciencia. [...] La ciencia no tiene, ni tendrá nunca, el mismo sentido de ser que el mundo percibido, por la razón de que solo es una determinación o explicación del mismo. (Merleau-Ponty, 1997, p. 8)

No se trata de deshacerse del saber científico, de sus métodos y resultados, sino más bien de colocarlo en su relación con el mundo de la vida. Esta es una advertencia muy pertinente para el campo de la educación: los que conviven en una escuela, los que se forman como ciudadanos son cuerpos vivos que se afectan mutuamente y que traen consigo, cada uno como sujetos vivos, una perspectiva específica de su propio mundo de vida.

Ahora bien, en un segundo argumento crítico Merleau-Ponty, retoma la máxima fenomenológica por excelencia, de "regresar a las cosas mismas" como primer principio epistemológico que permite rehabilitar la experiencia en la elaboración del conocimiento:

12 Los indicadores económicos se formulan en base a esta misma lógica, de esta forma, reducen la variable económica con promedios que, si bien no son espurios, no expresan en forma adecuada la complejidad. La renta per cápita, el PIB, incluso el índice de desarrollo humano (IDH) son muy aceptados en los rankings internacionales (ONU, 2018). Sin embargo, como es sabido tales indicadores ocultan que Chile es unos de los países más inequitativos del mundo (PNUD, 2018). 
Volver a las cosas mismas es volver a este mundo antes del conocimiento del que el conocimiento habla siempre, y respecto del cual toda determinación científica es abstracta, significativa y dependiente, como la geografía respecto del paisaje en el que aprendimos por primera vez qué era un bosque, un río o una pradera. (Merleau-Ponty, 1997, p. 9)

Esta vuelta a la experiencia no es de ninguna manera un llamado a la vuelta de un empirismo craso. La experiencia en este caso es la experiencia vivida, del espacio, del tiempo, un volver a la conciencia encarnada y la subjetividad. Es esta experiencia irreductible de ser ya-ahí en el mundo, la que ciencias habitualmente dejan fuera con sus abstracciones y definiciones. La experiencia del paisaje precede a la geografía.

Sobre la base de estos argumentos, queda claro cómo la perspectiva de los estándares y mediciones de la calidad, a pesar de su pretensión de conocimiento objetivo e incontrovertible, se revelan en sí abstracciones de supuestas determinaciones de un fenómeno dado, que no solamente siempre está en otra parte, sino que además precede en su manifestación y sentido a cualquier medida posible.

Por ejemplo, cuando la Agencia de la calidad (Chile, 2012,p. 13) muestra la fórmula matemática que recoge y expresa el indicador de la convivencia escolar ${ }^{13}$ podemos mirar y leer dicha fórmula de cálculo, desde la fenomenología, como una abstracción que puede ser comprendida si y sólo si se pone en perspectiva respecto del mundo de la vida; este precede y supera aquella fórmula, especialmente porque intenta dar cuenta de una expresión tan propia del mundo de la vida, como es la convivencia escolar.

No estamos planteando que aquella abstracción, expresada en la fórmula matemática, fuera priva de significado, sino que para su comprensión profunda y de su sentido es más pertinente y necesario reconocer su mediación desde el mundo de la vida. Esta mediación ha sido explorada y en la literatura que ha tratado explorar e investigar la educación desde la fenomenología (Gruenewald, 2003; Alerby, Hagström y Westman, 2014). Estas aproximaciones subrayan que el fenómeno educativo, particularmente en la escuela, se despliega en un tejido sólido y complejo hecho de cuerpos y sujetos conscientes que se desenvuelven en espacios y tiempos heterogéneos y diversos.

\section{CONCLUSIONES}

En este artículo hemos abordado las dimensiones de conocimiento implícitas en las políticas de accountability del sistema escolar y su pretensión de conocer y dar cuenta de la calidad educativa. Hemos llevado a cabo esta interrogación crítica mirando especialmente al caso chileno. En Chile la accountability de la calidad educativa se basa en un complejo aparato que ordena los colegios según categorías de desempeño, siguiendo procedimientos que incluyen pruebas estandarizados y

13 El documento de la Agencia de la Calidad mencionado da cuenta de la formulación matemática de cada uno de los otros indicadores de calidad, reportando las dimensiones de referencia y las fórmulas de cálculo. Por ejemplo, para el indicador de clima y convivencia escolar las dimensiones tomadas en cuenta son: ambiente organizado, ambiente de respeto y ambiente seguro. 
también otros indicadores de calidad relacionados a dimensiones de la experiencia escolar como la convivencia social y la participación ciudadana. El ordenamiento según categorías de desempeño implica una responsabilización de las escuelas por su quehacer y además prevé la posibilidad del cierre de los colegios en el caso de que se mantengan por un periodo de tres años en la categoría de desempeño baja.

Al final de este artículo podemos responder la pregunta que planteamos al comienzo respecto a qué problema de carácter epistemológico encierra la vinculación entre accountability y calidad educativa: pues bien, este nexo entre accountability y calidad educativa revela una concepción del conocimiento lineal que descansa en una pretensión de objetividad de las mediciones y de los estándares. Dicha epistemología se basa en el presupuesto que solo lo que se mide es real y por lo tanto se puede mejorar. Hemos mostrado, por el contrario, que los presupuestos de objetividad son puestos en tela de juicio por la ciencia física del siglo XX y por una epistemología de la incertidumbre y del mundo de la vida. El análisis epistemológico muestra una objetividad que se deshace y que se revela como algo siempre ligado a una dimensión subjetiva, por una parte, y por otra parte al mundo de la vida. En el uso político de test, indicadores y estándares se tiende a ponerlos entre paréntesis, o sencillamente se los omite.

En base de lo anterior, consideramos necesario considerar en una perspectiva diferente el uso de test, indicadores, y categorías de desempeño que constituyen los elementos medulares de la política de accountability con el propósito de asegurar la calidad educativa.

En particular, nos aproximamos a cuatro elementos que se pueden reflexionar y tomar en cuenta desde la discusión crítica que hemos desarrollado y que valen en general, así como para el caso chileno que hemos tomado aquí como referencia. Se trata de elementos que cobran particular relevancia a la luz de la suspensión de la aplicación del SIMCE en el año escolar 2020, debido a la situación de pandemia global por Covid 19:

- La necesidad de una discusión pública respecto de definiciones sustantivas de la calidad educativa. Se trata de mover el debate desde una racionalidad política que asocia la calidad a un resultado logrado en base de estándares, a una reconsideración de las condiciones de posibilidad de una buena experiencia educativa que ocurre en la escuela (Galioto, 2019);

- A la luz de las epistemologías de la complejidad que hemos esbozado, emerge la relevancia de buscar nuevos criterios respecto del cómo, dónde y cuándo dar cuenta de la calidad de la educación (Reinertsen,2016). Se trata de pensar modos que permitan una mejor síntesis y análisis del proceso y desempeño educativo en cuanto fenómenos intersubjetivos y atravesados por afectos, deseos y voluntades. Actualmente, dadas las dimensiones epistemológicas que hemos problematizado en el sistema de aseguramiento de la calidad educativa vigente en Chile, sería valioso y justo revisar las responsabilidades $\mathrm{y}$ consecuencias (que llegan incluso al cierre de los colegios) ${ }^{14}$;

14 De hecho, al final del 2019 en la comisión de Educación del senado se puso en tela de juicio este tipo de sanción legal. Algunos de los argumentos apuntaban justamente a la validez de las informaciones que luego llevan a ordenar los colegios por categorías de desempeño (Chile, 2019). 
- Si bien la calidad educativa puede ser medida, aunque sea como una aproximación a ella, sin embargo, es oportuno llevar a cabo esta operacionalización dentro de un contexto de accountability inteligente (Muller y Normand, 2013; O'Neill,2013) y no simplemente limitarse a constatar los déficits de un sistema desigual, que estandariza los resultados y los rendimientos de la propia inequidad, que estos mismos sistemas de medición generan y reproducen;

- La rendición de cuenta de la calidad educativa como herramienta valiosa de política educativa se puede beneficiar de una epistemología de la complejidad capaz de describir de otra forma ciertas dimensiones del fenómeno educativo. Al respecto Alexander $(2015$, p. 3) recuerda oportunamente la diferencia lógica encerrada en las nominalizaciones de la accountability: las medidas miden, los indicadores indican. Para ilustrar esta diferencia trae a colación el ejemplo de las nubes negras que son un indicador de lluvias probables y que, sin embargo, no proporcionan ninguna medida de cuánto podría llover. Análogamente, se trata de poder pensar indicadores pertinentes a los procesos pedagógicos y elaborar formas y procedimientos para recogerlos de manera adecuada.

Finalmente, se trata de mantener un umbral elevado de vigilancia crítica tanto hacia las premisas epistemológicas de la accountability en el ámbito educativo, como hacia el modo en que se utilizan los mecanismos de aseguramiento y evaluación de la calidad de los sistemas escolares, puesto que, al fin y al cabo, lo que está en juego en el proceso educativo escolar es lo que Hannah Arendt (1996) llama el amor al mundo y a su futuro.

\section{REFERENCIAS}

ALBUQUERQUE, P.; FERREIRA, A.; BARREIRA, C. Interdependência entre domínios na avaliação externa para a melhoria dos "resultados" em duas organizações escolares. Revista Brasileira de Educação, Rio de Janeiro, v. 25, e250022, 2020. https:// doi.org/10.1590/s1413-24782020250022

ALERBY, E.; HAGSTRÖM, E.; WESTMAN, S. The embodied classroom: a phenomenological discussion of the body and the room. Journal of Pedagogy, Berlín, v. 5, n. 1, p. 11-23, 2014. https://doi.org/10.2478/jped-2014-0001

ALEXANDER, R. Teaching and learning for all? The quality imperative revisited. International Journal of Educational Development, Paises Bajos, v. 40, p. 250-258, 2015. http://dx.doi.org/10.1016/j.ijedudev.2014.11.012

ARENDT, H. Entre el pasado y el futuro: ocho ejercicios sobre la reflexión política. Barcelona: Península, 1996.

AU, W. High-stakes testing and curricular control: a qualitative metasynthesis. Educational Researcher, Estados Unidos, v. 36, n. 5, p. 258-267, 2007. https://doi. org/10.3102/0013189X07306523 
BALL, S. The teacher's soul and the terrors of performativity. Journal of Education Policy, v. 18, n. 2, p. 215-228, 2003. https://doi.org/10.1080/0268093022000043065 BALL, S. Education, governance and the tyranny of numbers. Journal of Education Policy, v. 30, n. 3, 299-301, 2015. https://doi.org/10.1080/02680939.2015.1013271 BARTON, J.; KOPFMULLER, J. Santiago 2030: escenarios para la planificación estratégica. Santiago: RIL, 2016.

BREWER, C.; KNOEPPEL, R.; LINDLE, J. C. Consequential validity of accountability policy: public understanding of assessments. Educational Policy, Estados Unidos, v. 29, n. 5, p. 711-745, 2015. https://doi.org/10.1177/0895904813518099

CARRASCO, A. Mecanismos performativos de la institucionalidad educativa en Chile: pasos hacia un nuevo sujeto cultural. Observatorio Cultural, Santiago de Chile,n. 15,p.4-10,2013. CHEN, J.; TEO, T. Chinese school teachers' conceptions of high-stakes and low-stakes assessments: an invariance analysis. Educational Studies, England \& Wales, v. 46, n. 4, p. 458-475, 2020. https://doi.org/10.1080/03055698.2019.1599823

CHIANG, H. How accountability pressure on failing schools affects student achievement. Journal of Public Economics, v. 93, n. 9-10, p. 1045-1057, 2009. https:// doi.org/10.1016/j.jpubeco.2009.06.002

CHILE. Desarrollo personal y social: otros indicadores de calidad educativa. Descripción, metodología y resultados de los indicadores construidos en base a los Cuestionarios de Calidad y Contexto de la Educación. Chile: Agencia de la Calidad, 2012. Disponible en: http://archivos.agenciaeducacion.cl/Desarrollo_personal_social_ OIC_25_11.pdf. Acceso el: 14 abr. 2020.

CHILE. Formación ciudadana y participación en los establecimientos educacionales. Actitudes y percepciones de los estudiantes de II medio. Chile: Agencia de la Calidad, 2013. Disponible en: http://archivos.agenciaeducacion.cl/documentos-web/ Papers/2013_11_Formacion_Ciudadana_y_Participacion_en_los_establecimientos_ educacionales.pdf. Acceso el: 14 abr. 2020.

CHILE. Los indicadores de desarrollo personal y social en los establecimientos educacionales chilenos: una primera mirada. Chile: Agencia de la Calidad, 2015. Disponible en: http://archivos.agenciaeducacion.cl/estudios/Estudio_Indicadores_ desarrollo_personal_social_en_establecimientos_chilenos.pdf. Acceso el: 14 abr. 2020. CHILE. Informe técnico 2017. Indicadores de desarrollo personal y social medidos a través de cuestionarios. Chile: Agencia de la Calidad, 2017. Disponible en: http:// archivos.agenciaeducacion.cl/Informe_tecnico_IDPS_2017.pdf. Acceso el:14 abr. 2020. CHILE. Senado de Chile. Quieren evitar el cierre de escuelas por haber sido categorizadas en nivel "insuficiente" por la Agencia de Calidad de Educación. Chile: Senado de Chile, 2019. Disponible en: https://www.senado.cl/quieren-evitarel-cierre-de-escuelas-por-haber-sido-categorizadas-en/senado/2019-10-11/103832. html. Acceso el: 10 abr. 2020.

CIZEK, G. J.; ROSENBERG, S. L.; KOONS, H. Sources of validity evidence for educational and psychological tests. Educational and Psychological Measurement, Estados Unidos, v.68,n.3, p.397-412,2008.https://doi.org/10.1177/0013164407310130 
CLARKE, M. The sublime objects of education policy: quality, equity and ideology. Discourse: Studies in the Cultural Politics of Education, v. 35, n. 4, p. 584-598, 2014. https://doi.org/10.1080/01596306.2013.871230

CROCKER, L. Stakeholders in comprehensive validation of standards-based assessment. Educational Measurement: Issues and Practices, Estados Unidos, v. 21, n. 1, p. 5-6, 2002. https://doi.org/10.1111/j.1745-3992.2002.tb00079.x

CUMMING, J.; VAN DER KLEIJ, F.; ADIE, L. Contesting educational assessment policies in Australia.Journal of Education Policy, v. 34, n. 6, p. 836-857, 2019. https:// doi.org/10.1080/02680939.2019.1608375

DAVIS, A. High stakes testing and the structure of the mind: a reply to Randall curren. Journal of Philosophy of Education, Estados Unidos, v. 40, n. 1, p.1-16, 2006. https:// doi.org/10.1111/j.1467-9752.2006.00492.x

DAVIS, A. How far can we aspire to consistency when assessing learning? Ethics and Education, v. 8, n. 3, p. 217-228,2013. https://doi.org/10.1080/17449642.2013.876788 DAVIS, A. Is it really possible to test all educationally significant achievements with high levels of reliability? Ethics and Education, v. 10, n. 3, p. 372-379, 2015. https:// doi.org/10.1080/17449642.2015.1106032

DAVIS, A.; WINCH, C. Educational assessment on trial. Londres: Bloomsbury Academic, 2015.

DUBNICK, M. Accountability and the promise of performance: in search of the mechanisms. Public Performance \& Management Review, v. 27, n. 3, p. 376-417, 2005. EINSTEIN, A. Sobre la teoría de la relatividad especial y general.Madrid: Alianza, 2012. FALABELLA, A. The performing school: the effects of market $\&$ accountability policies. Education Policy Analysis Archives, Estados Unidos, v. 22, n. 70, p. 1-29, 2014. http://dx.doi.org/10.14507/epaa.v22n70.2014

FALABELLA, A. Do national test scores and quality labels trigger school self-assessment and accountability? A critical analysis in the Chilean context. British Journal of Sociology of Education, v.37, n. 5, p. 743-760,2016a. https://doi.org/10.1080/01425692.2014.976698 FALABELLA, A. ¿Qué aseguran las políticas de aseguramiento de la calidad? Un estudio de casos en distintos contextos escolares. Revista Estudios Pedagógicos, Valdivia, v. 42, n. 1, p. 107-126, 2016b. http://dx.doi.org/10.4067/S0718-07052016000100007

FALABELLA, A. La seducción por la hipervigilancia en la educación chilena: Simce, rendición de cuentas y Sistema de aseguramiento de la calidad (1979 a 2011). In: RUIZ, C.; HERRERA, F.; REYES, L. (eds.).La privatización de lo público en la educación escolar: el caso de Chile en perspectiva comparada. Santiago de Chile: LOM, 2019. p. 163-188. FIGLIO, D.; LADD, H. The economics of school accountability. International Encyclopedia of Education, Estados Unidos, p. 567-575, 2020. https://doi. org/10.1016/B978-0-12-815391-8.00042-2

FLORES, L.; MARINI, G. Dimensiones olvidadas de la reforma educativa hoy: la experiencia del espacio y el lugar en la escuela. In: CARRASCO, A.; FLORES, L. (eds.). De la reforma a la transformación: capacidades, innovaciones y regulación de la educación chilena. Santiago de Chile: CEPPE UC, 2018. p. 241-264. 
FLORES-GONZÁLEZ, L. Posiciones y orientaciones epistemológicas del paradigma de la complejidad. Cinta de Moebio, Santiago de Chile, v. 33, p. 195203, 2008. Disponible en: https://cintademoebio.uchile.cl/index.php/CDM/article/ view/25908/27222. Acceso en: 13 mar. 2020.

FLÓREZ, M. T. Análisis crítico de la validez del sistema de medición de la calidad de la educación (SIMCE). Santiago de Chile: Consejo Nacional de la Educación, 2013. Disponible en: https://www.cned.cl/proyecto-de-investigacion/analisis-criticode-la-validez-del-sistema-de-medicion-de-la-calidad-de-la. Acceso el: 13 abr. 2020.

FLÓREZ, M. T. Systems, ideologies and history: a three-dimensional absence in the study of assessment reform process. Assessment in Education: Principles, Policy \& Practice, v. 22, n. 1, p. 3-26, 2015. https://doi.org/10.1080/0969594X.2014.943153

FLÓREZ, M.T.; ROZAS, T. Accountability from a social justice perspective: criticism and proposals. Journal of Education Change, Estados Unidos, v. 21, p. 157-182, 2020. https://doi.org/10.1007/s10833-019-09361-3

FOCHLER, M.; DE RIJCKE, S. Implicated in the indicator game? An experimental debate. Engaging Science, Technology and Society, Estados Unidos, v. 3, p. 21-40, 2017. https://doi.org/10.17351/ests2017.108

GALIOTO, C. Schools without quality? Elements for a phenomenological exploration of the concept of quality in education. Phainomena, Ljubljana, v. 28, n. 110-111, p. 69-92, 2019. https://doi.org/10.32022/PHI28.2019.110-111.4

GAULON, R.; ROTHÉ, J. P. Séismes et sismologie: sismicité et tectonique des plaques. Encyclopædia Universalis, 2016. Disponible en: http://www.universalis.fr/ encyclopedie/seismes-et-sismologie-sismicite-et-tectonique-des-plaques/. Acceso el: 13 abr. 2020.

GREK, S. Governing by numbers: the PISA effect in Europe. Journal of Education Policy, v. 24, n. 1, p. 23-37, 2009. https://doi.org/10.1080/02680930802412669

GRUENEWALD, D. A. Foundations of place: a multidisciplinary framework for placeconscious education. American Educational Research Journal, Estados Unidos, v. 40, n. 3, p. 619-654, 2003. https://doi.org/10.3102/00028312040003619

HANKS, T.; KANAMORI, H. A moment magnitude scale. Journal of Geophysical Research, Estados Unidos, v. 84, n. B5, p. 2348-2350, 1979. https://doi.org/10.1029/ JB084iB05p02348

HARVEY, C. Making hollow men. Educational theory, Estados Unidos, v. 60, n. 2, p. 189-201, 2010. https://doi.org/10.1111/j.1741-5446.2010.00353.x

HEISENBERG, W. La imagen de la naturaleza en la física actual. Barcelona: Orbis, 1986.

HEMMER, L. M.; MADSEN, J.; TORRES, M. Critical analysis of accountability policy in alternative schools: implications for school leaders. Journal of Educational Administration, Inglaterra, v. 51, n. 5, p. 655-679, 2013. https://doi.org/10.1108/ JEA-01-2012-0002

KAUKO,J.; RINNE, R.; TAKALA, T. Politics of quality in education: a comparative study of Brazil, China, and Russia. Londres: Routledge, 2018. 
LACRUZ, A.; AMÉRICO, B.; CARNIEL, F. Indicadores de qualidade na educação: análise discriminante dos desempenhos na Prova Brasil. Revista Brasileira de Educação, Rio de Janeiro, v. 24, e240002, 2019. https://doi.org/10.1590/s141324782019240002

LEWIS, S.; LINGARD, B. The multiple effects of international large-scale assessment on education policy and research. Discourse Studies in the Cultural Politics of Education, v. 36, n. 5, p. 621-637, 2015. https://doi.org/10.1080/015 96306.2015.1039765

LINGARD, B.; MARTINO, W.; REZAI-RASHTI, G. Testing regimes, accountabilities and education policy: commensurate global and national developments. Journal of Education Policy, v. 28, n. 5, p. 539-556, 2013. https://doi.org/10.1080/0 2680939.2013.820042

LINGARD, B. et al. Globalizing educational accountabilities. Nueva York: Routledge, 2015.

MATURANA, H. La realidad: ¿ cobjetiva o construida? Barcelona: Anthropos, 1995.

MATURANA, H. La objetividad: un argumento para obligar. Madrid: Paidós, 2020. MERLEAU-PONTY, M. Fenomenología de la percepción. Barcelona: Ediciones Península, 1997.

MULLER, F.; NORMAND, R. Ecole: la grande transformation? Les clés de la réussite. Strasbourg: ESF Science Humaines, 2013. Disponible en: https://esfscienceshumaines.fr/pedagogie/125-ecole-la-grande-transformation-.html. Acceso el: 14 abr. 2020.

NORMAND, R. La mesure de l'école : politique des standards et management par la qualité. Cahiers de la Recherche sur L'éducation et les Savoirs Hors-série, n. 1, p. 67-82, 2005. Disponible en: http://journals.openedition.org/cres/1911. Acceso el: 24 mar. 2020.

OLIVA, M. A. Política educativa chilena 1965-2009: ¿Qué oculta esa trama? Revista Brasileira de Educação, Rio de Janeiro, v. 15, n. 44, p. 311-328, 2010. https://doi. org/10.1590/S1413-24782010000200008

O'NEILL, O. Intelligent accountability in education. Oxford Review of Education, v. 39, n. 1, p. 4-16, 2013. https://doi.org/10.1080/03054985.2013.764761

ORGANIZACIÓN DE LAS NACIONES UNIDAS (ONU). Chile mantiene primer lugar en Desarrollo Humano en América Latina y el Caribe e igualdad de género es su mayor desafío. ONU, 2018. Disponible en: https://www.cl.undp.org/ content/chile/es/home/presscenter/pressreleases/2018/chile-mantiene-primer-lugaren-desarrollo-humano-entre-paises-de.html. Acceso el: 11 out. 2021.

ORTÍZ, I. En torno a la validez del Sistema Medición de la Calidad de la Educación. Estudios Pedagógicos, Valdivia, v. 38, n. 2, p. 355-373, 2012.http://dx.doi.org/10.4067/ S0718-07052012000200022

OZGA,J. Accountability as a policy technology: accounting for education performance in Europe. International Review of Administrative Sciences, Estados Unidos, v. 79, n. 2, p. 292-309, 2013. https://doi.org/10.1177/0020852313477763 
PENROSE, R. El camino a la realidad: una guía completa de las leyes del Universo. Barcelona: Debate, 2007.

PIKETTY,T. El capital en el siglo XXI. Madrid: Fondo de Cultura Económica, 2014. PIKETTY,T.; ZUCMAN, G. Capital is back: wealth-income ratios in rich countries, 1700-2010. Paris: École d'Économie de Paris, 2013.

POWER, M. The audit society: rituals of verification. Oxford: Oxford University Press, 1997.

PROGRAMA DE LAS NACIONES UNIDAS PARA EL DESARROLLO (PNUD). Desigualdad Regional En Chile. PNUD, 2018. Disponible en: https:// www.estudiospnud.cl/informes-desarrollo/desiguales-origenes-cambios-y-desafiosde-la-brecha-social-en-chile/. Acceso el: 11 out. 2021.

RAMOS, C. Dispositivo de evaluación y gubernamentalidad del sistema educacional: entretejimiento de ciencia social y poder. Cinta Moebio, Santiago de Chile, n. 61, p. 41-55, 2018. http://doi.org/10.4067/S0717-554X2018000100041

REINERTSEN, A. B. A Phaedrus baroque art of maintenance or constant fabulating qualia becoming quality. Contemporary Issues in Early Childhood, Estados Unidos, v. 17, n. 1, p. 112-123, 2016. https://doi.org/10.1177/1463949115627909

ROSENKVIST, $M$. Using student test results for accountability and improvement: a literature review. OECD Education Working Papers, n. 54, 2010.

\section{SANMARTÍN,E.; CARRASCO,A.Clasificación de escuelasenla nuevainstitucionalidad} educativa: contribución de modelos de valor agregado para una responsabilización justa. Santiago de Chile: Centro de Políticas Públicas, 2012. n. 53. Disponible en: https:// politicaspublicas.uc.c1/wp-content/uploads/2015/02/descargar-clasificacion-de-escuelas-enla-nueva-institucionalidad-educativa.pdf. Acceso el: 22 ene. 2020.

SELLAR, S. A feel for numbers: affect, data and education policy. Critical Studies in Education, v. 56, n. 1, p. 131-146, 2015a. https://doi.org/10.1080/17508487.2015 .981198

SELLAR, S. Transparency and opacity: levinasian reflections on accountability in Australian schooling. Educational Philosophy and Theory, v. 47, n. 2, p. 118-132, 2015b. https://doi.org/10.1080/00131857.2013.793924

SELLAR, S.; LINGARD, B. The OECD and the expansion of PISA: new global modes of governance in education. British Educational Research Journal, Estados Unidos, v. 40, n. 6, p. 917-936, 2014. https://doi.org/10.1002/berj.3120

SELLAR, S.; LINGARD, B. Looking East: Shanghai, PISA 2009 and the reconstitution of reference societies in the global education policy field. Comparative Education, v. 49, n. 4, p. 464-485, 2013. https://doi.org/10.1080/03050068.2013.770943

SELWYN, N. "There's so much data": exploring the realities of data-based school governance. European Educational Research Journal, Estados Unidos, v. 15, n. 1, p. 54-68, 2015. https://doi.org/10.1177/1474904115602909

SUSPITSYNA, T. Accountability in American education as a rhetoric and a technology of governmentality.Journal of Educational Policy, v. 25, n. 5, p. 567-586, 2010. https:// doi.org/10.1080/02680930903548411 
VERGER, A.; FONTDEVILA, C.; PARCERISA, L. Reforming governance through policy instruments: How and to what extent standards, tests and accountability in education spread worldwide. Discourse: Studies in the Cultural Politics of Education, v. 40, n. 2, p. 248-270, 2019. https://doi.org/10.1080/01596306.2019.1569882

YDESEN, C. Educational testing as an accountability measure: drawing on twentieth-century Danish history of education experiences. Paedagogica Historica: International Journal of the History of Education, v. 49, n. 5, p. 716-733, 2013. https://doi.org/10.1080/00309230.2013.815235

\section{SOBRE LOS AUTORES}

Carmelo Galioto-Allegra es investigador postdoctoral en la Universidad de O'Higgins (Chile).

E-mail: carmelo.galioto@uc.cl

Luís Manuel Flores-Gonzalez es doctor en filosofía en la Universidad Católica de Lovaina (Bélgica). Profesor de la Pontificia Universidad Católica de Chile (Chile).

E-mail:1mflores@uc.cl

Conflictos de interés: Los autores declaran no tener ningún interés comercial o asociativo que constituya conflicto de interés con relación al manuscrito.

Financiamiento: El estúdio no recibió financiamiento.

Contribuiciones de los autores: Administración del Proyecto, Analisis Formal, Conceptualización, Curadoría de Data, Escrita - Primera Redacción, Escrita - Revisión y Edición: Galioto-Allegra, C.; Flores-Gonzalez, L. M.

Recibido el 5 de septiembre de 2020 Aprobado el 2 de marzo de 2021 